\title{
Systematic Study of Survival Probability of Excited Superheavy Nuclei
}

\author{
Cheng-Jun XIA ${ }^{1}$, Bao-Xi SUN ${ }^{1}$, En-Guang ZHAO ${ }^{2,3} \&$ Shan-Gui ZHOU ${ }^{2,3 *}$ \\ ${ }^{1}$ College of Applied Sciences, Beijing University of Technology, Beijing 100124, China; \\ ${ }^{2}$ Institute of Theoretical Physics, Chinese Academy of Sciences, Beijing 100190, China; \\ ${ }^{3}$ Center of Theoretical Nuclear Physics, National Laboratory of Heavy Ion Accelerator, Lanzhou 730000, China
}

Received November 23, 2010; accepted December 20, 2010

\begin{abstract}
The stability of excited superheavy nuclei (SHN) with $100 \leqslant Z \leqslant 134$ against neutron emission and fission is investigated by using a statistical model. In particular, a systematic study of the survival probability against fission in the $1 \mathrm{n}$-channel of these $\mathrm{SHN}$ is made. In present calculations the neutron separation energies and shell correction energies are consistently taken from the calculated results of the finite range droplet model which predicts an island of stability of SHN around $Z=115$ and $N=179$. It turns out that this island of stability persists for excited SHN in the sense that the calculated survival probabilities in the 1n-channel of excited SHN at the optimal excitation energy are maximized around $Z=115$ and $N=179$. This indicates that the survival probability in the $1 \mathrm{n}$-channel is mainly determined by the nuclear shell effects.
\end{abstract}

survival probability, superheavy elements, island of stability, neutron emission, fission

PACS: 24.10.Pa, 24.60.Dr, 25.85.-w, 27.90.+b, 21.10.-k

\section{Introduction}

The importance of quantum shell effects in stabilizing heavy nuclei was realized and the existence of superheavy elements was predicted in 1960s [1] In experiment, via cold fusion reactions, superheavy elements with $102 \leqslant Z \leqslant 112$ have been synthesized in GSI [4] and that with $Z=113$ in RIKEN [F]. Superheavy elements with $Z$ up to 118 have also been synthesized with hot fusion reactions in Dubna [6,7]. Theoretical investigations of SHN are focused both on the structure and decay properties [8 [13] and on the synthesis mechanism [14 19].

A superheavy nucleus in its ground state can decay via spontaneous fission, $\alpha$-decay, $\beta$-decay, etc.. As a crucial ingredient of the existence of the island of stability, the stability of superheavy nuclei in their ground states has been studied extensively since the existence of superheavy elements was predicted [20]. The stability of an excited superheavy nuclei is also an important issue. On the one hand, it helps us in understanding the stability behavior of a superheavy nucleus against excitation, e.g., the attenuation of the shell effects with temperature. On the other hand, the survival probability $W_{\text {sur }}$ of an excited compound nucleus against processes in which the charge number is changed is directly related with the stability of an excited superheavy nucleus in various channels. The survival probability $W_{\text {sur }}$ is an important factor in the the study of synthesis mechanism of superheavy elements. Presently most of the calculations are focused on the stability of superheavy compound nuclei formed in cold and/or hot fusion reactions 2124 .

In order to study the influence of the shell effects on the stability of superheavy nuclei with excitation, we carry out a systematic investigation of the stability of excited superheavy nuclei. In the present work, the stability of excited superheavy nuclei with $100 \leqslant Z \leqslant 134$ against neutron emission and fission is studied by using a statistical model. As an example, we present a systematic study of the survival probability against fission in the $1 \mathrm{n}$-channel of these superheavy nuclei. It is well known that there are quite different predictions of the shell structure of superheavy nuclei from different models. As a consequence, the next proton magic number after 82 could be 114, 120, 126, etc.. In this paper, the properties of superheavy nuclei, e.g., the neutron separation energies and shell correction energies, are consistently taken from predictions of the finite range droplet model [25].

The paper is organized as follows. In Sec. 目, the formalism of the statistical model is briefly sketched. The results and discussions are presented in Sec. B. Finally a summary is given in Sec. 4 .

*Email: sgzhou@itp.ac.cn 


\section{Formalism}

An excited superheavy compound nucleus can decay via fission or emitting photon(s), neutron(s), proton(s) or light-charged particle(s) like $\alpha$-particle. Among all these channels, the most favorable ones are fission and neutron(s) emission. In the present study, we mainly focus on these two channels.

The decay width for the neutron emission of a compound nucleus with excitation energy $E^{*}$ and spin $J$ is calculated as [26]

$$
\Gamma_{\mathrm{n}}\left(E^{*}, J\right)=\frac{2 m_{\mathrm{n}} R^{2}}{\pi \hbar^{2}} \int_{0}^{E^{*}-S_{\mathrm{n}}} \frac{\rho\left(E^{*}-S_{\mathrm{n}}-\varepsilon_{\mathrm{n}}, J\right)}{\rho\left(E^{*}, J\right)} \varepsilon_{\mathrm{n}} d \varepsilon_{\mathrm{n}},
$$

where $m_{\mathrm{n}}$ is the neutron mass, $S_{\mathrm{n}}$ is the neutron separation energy, $R=r_{0} A^{1 / 3}$ is the radius of the compound nucleus with $r_{0}=1.20 \mathrm{fm}$, and $\rho\left(E^{*}, J\right)$ is the level density which is discussed later.

The fission width can be calculated with the Bohr-Wheeler formula as 27

$$
\Gamma_{\mathrm{f}}\left(E^{*}, J\right)=\frac{1}{2 \pi} \int_{0}^{E^{*}-B_{\mathrm{f}}} \frac{\rho_{\text {s.d. }}\left(E^{*}-B_{\mathrm{f}}-\varepsilon_{\mathrm{f}}, J\right)}{\rho_{\text {s.d. }}\left(E^{*}, J\right)} T_{\mathrm{f}}\left(\varepsilon_{\mathrm{f}}\right) d \varepsilon_{\mathrm{f}},
$$

where $B_{\mathrm{f}}$ is the fission barrier, $\rho_{\text {s.d. }}\left(E^{*}, J\right)$ is the level density at the saddle point, and $T_{\mathrm{f}}\left(\varepsilon_{\mathrm{f}}\right)$ is the barrier transmission probability,

$$
T_{\mathrm{f}}\left(\varepsilon_{\mathrm{f}}\right)=\left\{1+\exp \left[-\frac{2 \pi \varepsilon_{\mathrm{f}}}{\hbar \omega_{\text {s.d. }}}\right]\right\}^{-1},
$$

with the barrier width $\hbar \omega_{\text {s.d. }}=2.2 \mathrm{MeV}[23]$. The fission barrier height including the washing out effect of shell effects with the excitation energy $E^{*}$ and spin $J$ is given as [28]

$$
B_{\mathrm{f}}\left(E^{*}, J\right)=B_{\mathrm{f}}^{\mathrm{Mac}}+B_{\mathrm{f}}^{\mathrm{Mic}} \exp \left(-\frac{E^{*}}{E_{\mathrm{D}}}\right)-\left(\frac{\hbar^{2}}{2 \mathcal{J}_{\text {g.s. }}}-\frac{\hbar^{2}}{2 \mathcal{J}_{\text {s.d. }}}\right) J(J+1),
$$

where $B_{\mathrm{f}}^{\text {Mac }}$ is the macroscopic part of the fission barrier height of the compound nucleus [29]. Under the assumption that the shell correction energy at the saddle point is negligible, the microscopic part of the fission barrier height $B_{\mathrm{f}}^{\text {Mic }}=-E_{\mathrm{Mic}}$ with $E_{\text {Mic }}$ the shell correction energy in the ground state. There are several expressions for the damping parameter $E_{\mathrm{D}}[30,31]$ and we take $E_{\mathrm{D}}=5.48 A^{1 / 3} /\left(1+1.3 A^{-1 / 3}\right)[30$. The moments of inertia of the compound nucleus in its ground state and at the saddle point are calculated as

$$
\mathcal{J}_{\text {g.s.(s.d.) }}=\frac{2}{5} M R^{2}\left(1+\beta_{2}^{\text {g.s.(s.d.) }} / 3\right) \text {. }
$$

The level density is calculated from the Fermi-gas model according to Ref. [32],

$$
\rho\left(E^{*}, J\right)=\frac{2 J+1}{24 \sqrt{2} \sigma^{3} a^{1 / 4}\left(E^{*}-\delta\right)^{5 / 4}} \exp \left[2 \sqrt{a\left(E^{*}-\delta\right)}-\frac{(J+1 / 2)^{2}}{2 \sigma^{2}}\right],
$$

with

$$
\sigma^{2}=6 \bar{m}^{2} \sqrt{a\left(E^{*}-\delta\right)} / \pi^{2}, \quad \bar{m}^{2} \approx 0.24 A^{2 / 3},
$$

where the level density parameter $a_{\text {s.d. }}=1.1 A / 12 \mathrm{MeV}^{-1}$ at the saddle point and $a=A / 12 \mathrm{MeV}^{-1}$ in other cases. The pairing correction $\delta=12 / \sqrt{A} \mathrm{MeV}, 0$ and $-12 / \sqrt{A} \mathrm{MeV}$ for even-even, odd-even and odd-odd nuclei, respectively [24].

The survival probability in the $1 \mathrm{n}$-channel is calculated by

$$
W_{\text {sur }}\left(E^{*}, J\right)=P_{1 \mathrm{n}}\left(E^{*}, J\right) \frac{\Gamma_{\mathrm{n}}\left(E^{*}, J\right)}{\Gamma_{\mathrm{n}}\left(E^{*}, J\right)+\Gamma_{\mathrm{f}}\left(E^{*}, J\right)},
$$

where the realization probability for $1 \mathrm{n}$-emission reads 21

$$
P_{1 \mathrm{n}}\left(E^{*}, J\right)=\exp \left[-\frac{\left(E^{*}-S_{n}-2 T\right)^{2}}{2 \Sigma^{2}}\right] .
$$

The nuclear temperature $\left.T=\left[1+\sqrt{1+4 a E^{*}}\right)\right] / 2 a, \Sigma=2.2 \mathrm{MeV}$ and $a$ is the level density parameter. 


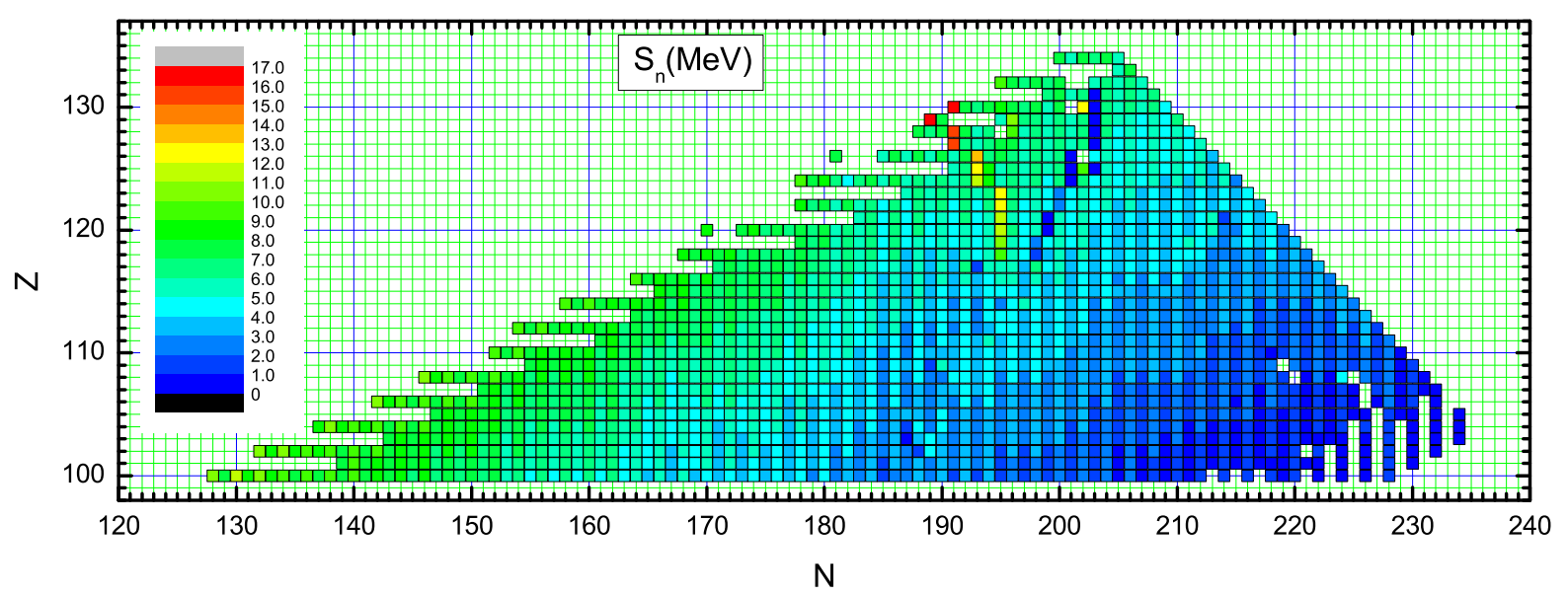

Figure 1: The neutron separation energies of superheavy nuclei with $100 \leqslant Z \leqslant 134$ predicted by the finite range droplet model [25].

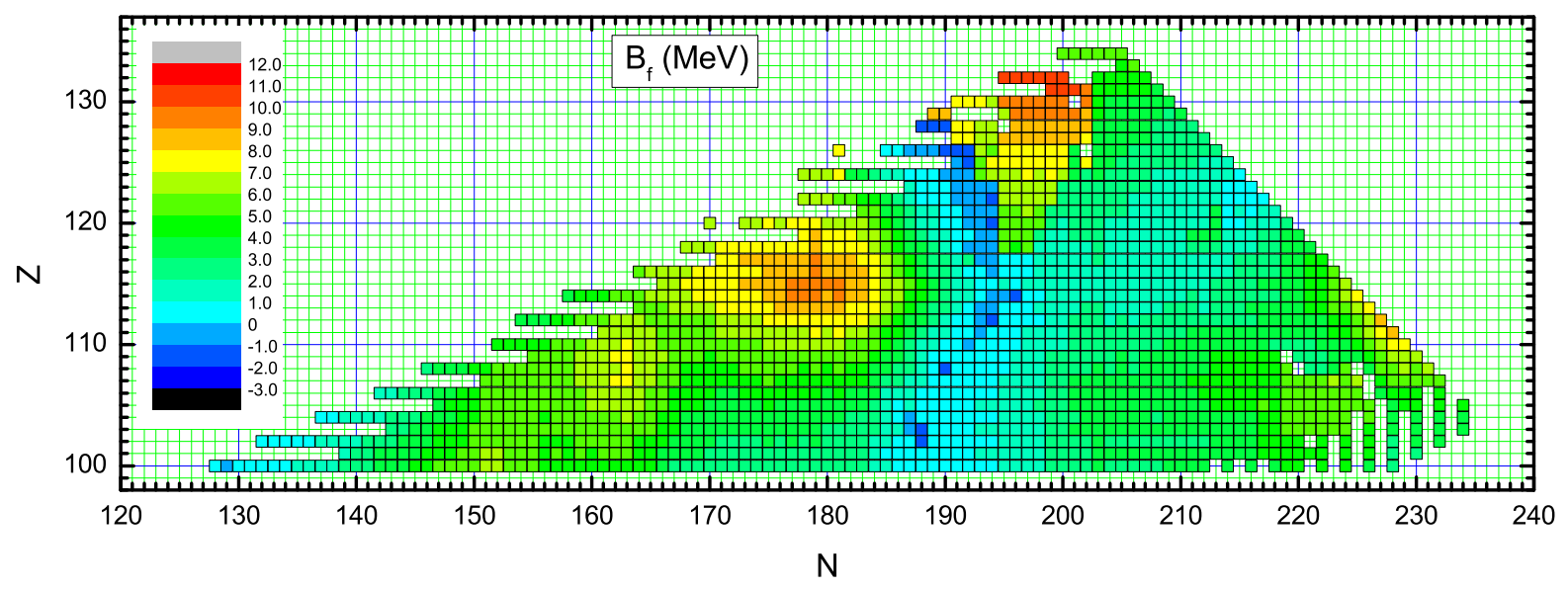

Figure 2: The fission barriers of superheavy nuclei with $100 \leqslant Z \leqslant 134$ at $E^{*}=0$ and $J=0$ calculated from Eq. (円) where the shell correction energies are taken from the finite range droplet model [25].

\section{Results and discussions}

For the superheavy nuclei, there are different predictions of ground state and saddle point properties. Since both the neutron emission and fission processes are connected closely to the shell structure, in order to study the influence of the shell effects on the stability of superheavy nuclei with excitation, one should take the nuclear property parameters for calculating the neutron emission and fission consistently from one single model. In the present work, the properties of superheavy nuclei with $100 \leqslant$ $Z \leqslant 134$ are taken from predictions of the finite range droplet model [25].

One of the crucial parameters for calculating the width of neutron emission is the neutron separation energy. Figure 1 shows the neutron separation energies of superheavy nuclei with $100 \leqslant Z \leqslant 134$ from the finite range droplet model [25]. Only those nuclei within the proton drip line and the neutron drip line are included. One finds several common features in Fig. 1. First, the neutron separation energy decreases with neutron number. Second, there is clearly odd-even effects in the neutron separation energy. With these values for the neutron separation energies and level density parameters given in Sec. 2 , the width of neutron emission is calculated from Eq. (1).

The fission barrier heights of superheavy nuclei with $100 \leqslant Z \leqslant 134$ at $E^{*}=0$ and $J=0$ are shown in Fig. 目 with the shell correction energies taken from the finite range droplet model [25]. The fission barrier height of a superheavy nucleus is mainly determined by the shell correction energy because the macroscopic part $B^{\mathrm{Mac}}$ is quite small. According to Eq. (4), a larger negative shell correction energy results in a higher fission barrier. The finite range droplet model predicts that the island 


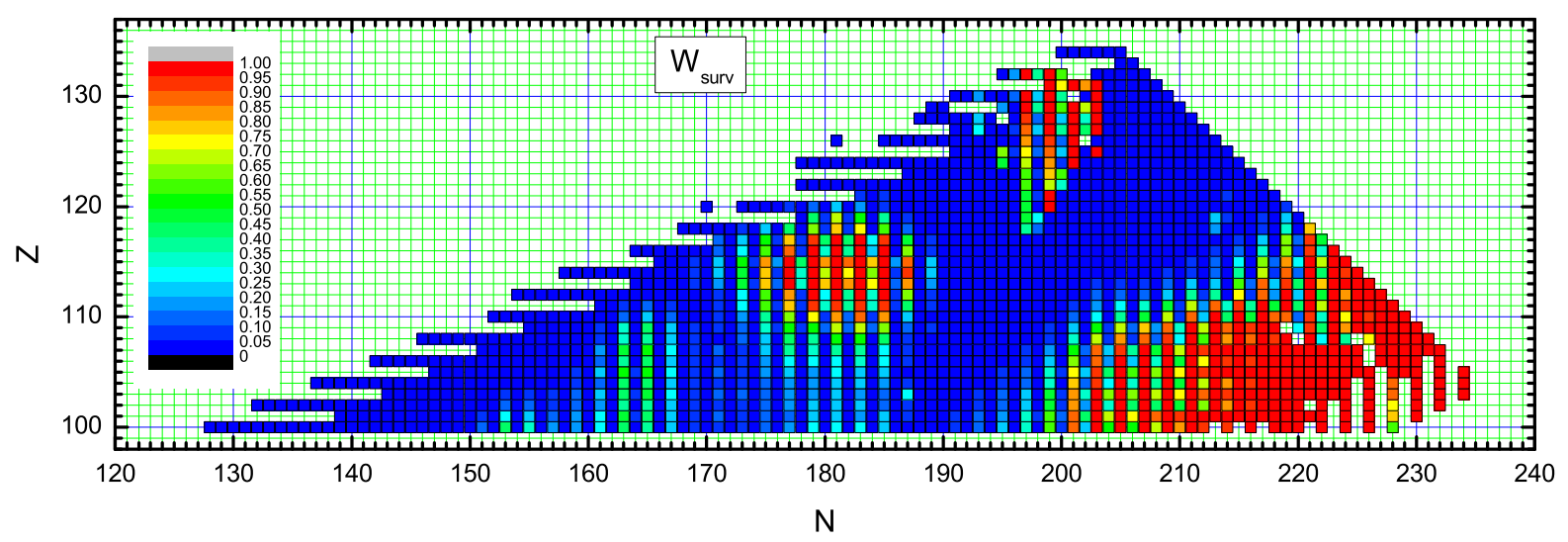

Figure 3: The survival probability against fission of excited superheavy nuclei in the 1 n-channel with $100 \leqslant Z \leqslant 134$ at the optimal excitation energy and $J=0$.

of stability centers around $Z=115$ and $N=179$ due to the quantum shell effects. Correspondingly, there is a region with higher fission barrier in Fig. 目 around $Z=115$ and $N=179$. Meanwhile the deformed sub-shells at $Z=108$ and $N=162 \sim 164$ also manifest themselves with fission barriers as high as about $7 \sim 8 \mathrm{MeV}$. In addition, there is another mass region around $Z=130$ and $N=198$ in which the superheavy nuclei also have very high fission barriers. There is a roughly vertical band around $N=190$ in which the fission barrier heights of the nuclei are negative. This means that these nuclei do not exist according to the finite range droplet model.

The survival probability of an excited superheavy nucleus with $J=0$ in the 1 -channel calculated from Eq. (8), as a function of the excitation energy $E^{*}$, shows an anti-parabolic shape. We take the maximal value in the $W_{\text {sur }} \sim E^{*}$ curve for each superheavy nucleus and define the corresponding $E^{*}$ as the optimal excitation energy.

In Fig. 3 the survival probability of excited superheavy nuclei with $100 \leqslant Z \leqslant 134$ at the optimal excitation energies are given. There are two islands with larger survival probability in Fig. B which roughly correspond to the islands of superheavy nuclei with higher fission barriers as shown in Fig. 2 . This indicates that for a superheavy nucleus in these two mass regions, the fission width is quite small due to the high fission barrier. In the very neutron-rich region, the neutron separation energy is small which results in a large neutron emission width. Therefore the survival probability in the 1n-channel becomes larger in the very neutron-rich region if one only includes the fission and neutron emission processes. For these nuclei, other decay channels should be taken into account. The survival probability of superheavy nuclei in the 1 n-channel shows clear odd-even effects which is mainly from the odd-even effects in the neutron separation energy.

From the above discussions, one can conclude that the shell effects also plays important roles in the stability behavior and survival probability of excited superheavy nuclei. Therefore nuclear parameters such as the separation energy and fission barriers from models which predict different shell structures in the region of superheavy nuclei may give very different survival probability. This may also result in different trends in the evaporation residue cross section as a function of the proton number in the experimentally unknown mass region.

Besides the neutron separation energy and the fission barrier which are directly related to the shell effects, there are some other parameters which are also very important in the calculation of the survival probability. For example, the parameters for the level density influence the decay width very much [24]. Therefore, not only accurate nuclear properties such as the neutron separation energy and the fission barrier, but also a proper form of the level density are needed for an accurate prediction of the survival probability of an excited superheavy nucleus.

\section{Summary}

In order to study the influence of the shell effects on the stability of superheavy nuclei with excitation, the stability of excited superheavy nuclei with $100 \leqslant Z \leqslant 134$ against neutron emission and fission is studied by using a statistical model.

As an example, we present a systematic study of the survival probability in the 1n-channel of these superheavy nuclei with $J=0$. In this work, the properties of superheavy nuclei including the neutron separation energies and shell correction energies are consistently taken from predictions of the finite range droplet model. The islands of stability of superheavy nuclei in their ground state, e.g., the one around $Z=115$ and $N=179$, persist for excited superheavy nuclei in the sense that the calculated 
survival probabilities in the 1n-channel of excited superheavy nuclei on these islands are maximized. This indicates that the survival probability is mainly determined by the nuclear shell effects.

Finally it should be emphasized that the decay widths in different channels and the survival probabilities of superheavy nuclei are very sensitive to the nuclear properties such as the separation energy, the ground state and saddle point deformations, the fission barriers, and the level density, etc.. Therefore in the study of the decay properties of superheavy nuclei, reliable predictions of these properties from nuclear models is highly desirable.

We thank G. G. Adamian, N. V. Antonenko, A. Nasirov and A. S. Zubov for fruitful discussions and suggestions. This work has been supported by the National Natural Science Foundation of China (Grant Nos. 10705014, 10775012, 10875157, 10975100, and 10979066), Major State Basic Research Development Program of China (Grant No. 2007CB815000), and Knowledge Innovation Project of Chinese Academy of Sciences (Grant Nos. KJCX2-EW-NO1 and KJCX2YW-N32). The computation of this work was supported by Supercomputing Center, CNIC of CAS.

1 Myers W D, Swiatecki W J. Nuclear masses and deformations. Nucl Phys, 1966, 81: 1-60

2 Sobiczewski A, Gareev F, Kalinkin B. Closed shells for $Z>82$ and $N>126$ in a diffuse potential well. Phys Lett, 1966, 22: 500-502

3 Meldner H. Predictions of new magic regions and masses for super-heavy nuclei from calculations with realistic shell model single particle Hamiltonian. Arkiv Fysik, 1967, 36: 593-598

4 Hofmann S, Muenzenberg G. The discovery of the heaviest elements. Rev Mod Phys, 2000, 72: 733-767

5 Morita K, Morimoto K, Kaji D, et al. Experiment on the Synthesis of Element 113 in the Reaction ${ }^{209} \mathrm{Bi}\left({ }^{70} \mathrm{Zn}, \mathrm{n}\right)^{278} 113$. J Phys Soc Jpn, 2004, 73 : 2593-2596

6 Oganessian Y. Heaviest nuclei from ${ }^{48}$ Ca-induced reactions. J Phys G: Nucl Part Phys, 2007, 34: R165-R242

7 Oganessian Y T, Abdullin F S, Bailey P D, et al. Synthesis of a New Element with Atomic Number Z = 117. Phys Rev Lett, 2010, 104: 142502-4

8 Lalazissis G A, Sharma M M, Ring P, et al. Superheavy nuclei in the relativistic mean-field theory. Nucl Phys A, 1996, 608: 202-226

9 Rutz K, Bender M, Burvenich T, et al. Superheavy nuclei in self-consistent nuclear calculations. Phys Rev C, 1997, 56: 238-243

10 Long W, Meng J, Zhou S G. Structure of the new nuclide ${ }^{259} \mathrm{Db}$ and its alpha-decay daughter nuclei. Phys Rev C, 2002, 65: 047306-4

11 Ren Z, Chen D H, Tai F, et al. Ground state properties of odd-Z superheavy nuclei. Phys Rev C, 2003, 67: 064302-12

12 Zhang W, Meng J, Zhang S, et al. Magic numbers for superheavy nuclei in relativistic continuum Hartree-Bogoliubov theory. Nucl Phys A, 2005, 753: 106-135

13 Zhang H, Zuo W, Li J, et al. Alpha decay half-lives of new superheavy nuclei within a generalized liquid drop model. Phys Rev C, 2006, 74: 017304-4

14 Li W, Wang N, Li J F, et al. Fusion probability in heavy-ion collisions by a dinuclear-system model. Europhys Lett, 2003, 64: 750-756

15 Zhang F S, Feng Z Q, Jin G M. Importance of shell correction energy on synthesis of superheavy nuclei. Int J Mod Phys E, 2006, 15: 1601-1611

16 Feng Z Q, Jin G M, Li J Q, et al. Formation of superheavy nuclei in cold fusion reactions. Phys Rev C, 2007, 76: 044606-9

17 Shen C, Abe Y, Boilley D, et al. Isospin dependence of reactions ${ }^{48} \mathrm{Ca}+{ }^{243-251} \mathrm{Bk}$. Int J Mod Phys E, 2008, 17: 66-79

18 Liu Z H, Bao J D. Synthesis of superheavy element 120 via ${ }^{50} \mathrm{Ti}+{ }^{A} \mathrm{Cf}$ hot fusion reactions. Phys Rev C, 2009, 80: 054608-5

19 Zagrebaev V, Greiner W. New ideas on the production of heavy and superheavy neutron rich nuclei. Nucl Phys A, 2010, 834: 366c-369c

20 Nilsson S G, Tsang C F, Sobiczewski A, et al. On the nuclear structure and stability of heavy and superheavy elements. Nucl Phys A, 1969, 131: 1-66

21 Adamian G G, Antonenko N V, Ivanova S P, et al. Analysis of survival probability of superheavy nuclei. Phys Rev C, 2000, 62: 064303-6

22 Zubov A S, Adamian G G, Antonenko N V, et al. Survival probability of superheavy nuclei. Phys Rev C, 2002, 65: 024308-10

23 Zubov A S, Adamian G G, Antonenko N V, et al. Survival probabilities of superheavy nuclei based on recent predictions of nuclear properties. Eur Phys J A, 2005, 23: 249-256

24 Zubov A, Adamian G, Antonenko N. Application of statistical methods for analysis of heavy-ion reactions in the framework of a dinuclear system model. Phys Part Nucl, 2009, 40: 847-889

25 Möller P, Nix J R, Myers W D, et al. Nuclear Ground-State Masses and Deformations. At Data Nucl Data Tables, 1995, 59: 185-381

26 Feng Z Q, Jin G M, Fu F, et al. Production cross sections of superheavy nuclei based on dinuclear system model. Nucl Phys A, 2006, 771: 50-67

27 Bohr N, Wheeler J A. The Mechanism of Nuclear Fission. Phys Rev, 1939, 56: 426-450

28 Li W, Wang Z, Xu H, et al. Odd-Even Effects of the Survival Probability for Superheavy Compound Nuclei. Chin Phys Lett, 2004, 21: 636-639

29 Dahlinger M, Vermeulen D, Schmidt K H. Empirical saddle-point and ground-state masses as a probe of the droplet model. Nucl Phys A, 1982, 376: 94-130

30 Ignatyuk A V, Itkis M G, Okolovich V N, et al. Fission of pre-actinide nuclei - excitation-functions for ( $\alpha$,f) reaction. Sov J Nucl Phys, 1975, 21: 612-621

31 Schmidt K H, Morawek W. The conditions for the synthesis of heavy nuclei. Rep Prog Phys, 1991, 54: 949-1003

32 Ignatyuk A V, Istekov K K, Smirenkin G N. Role of Collective Effects in the Systematics of Nuclear-level Densities. Sov J Nucl Phys, 1979, 29: 450-454 